\title{
INFINITE MEASURE PRESERVING TRANSFORMATIONS WITH "MIXING"
}

BY S. KAKUTANI AND W. PARRY ${ }^{1}$

Communicated by W. S. Massey, June 24, 1963

1. Introduction. It is well known that a transformation $T$ which preserves a finite measure has the mixing property

$$
\begin{gathered}
T^{(k)}=T \times T \times \cdots \times T(k \text { times, } k \geqq 2) \text { is ergodic } \\
\quad \text { if and only if } T \text { is weakly mixing [1]. }
\end{gathered}
$$

The purpose of this note is to give, for each positive integer $k$, an example of a transformation $T$ which preserves a $\sigma$-finite infinite measure with the property,

$$
T^{(k)} \text { is ergodic but } T^{(k+1)} \text { is not ergodic. }
$$

We also give an example of a transformation $T$ which preserves a $\sigma$-finite infinite measure with the property

$$
T^{(k)} \text { is ergodic for each } k=1,2, \cdots .
$$

A transformation $T$ with property (1.2) is said to have ergodic index $k$ and a transformation $T$ with property (1.3) is said to have infinite ergodic index. For completeness, we say that a nonergodic transformation has zero ergodic index.

Thus, for each $k=0,1,2, \cdots, \infty$, infinite measure preserving transformations exist with ergodic index $k$, unlike finite measure preserving transformations which assume ergodic indices $0,1, \infty$ only.

The examples are taken from Gillis [2], and are Markov transformations derived from "centrally biased random-walks." Let

2. Markov transformations preserving a $\sigma$-finite infinite measure.

$$
P=\|p(i, j)\|, \quad i, j=0, \pm 1, \pm 2, \cdots
$$

be a stochastic matrix with only one ergodic class, i.e.,

$$
p(i, j) \geqq 0, \quad \sum_{j=-\infty}^{\infty} p(i, j)=1,
$$

and for each $(i, j)$ there exists $n>0$ for which $p^{n}(i, j)>0$ where $P^{n}=\left\|p^{n}(i, j)\right\|$. Assume also that there exists a left eigenvector

\footnotetext{
${ }^{1}$ Research supported in part by NSF G-25222.
} 
$\Lambda=\{\lambda(i)\}$ (with eigenvalue one) with positive entries such that

$$
\sum_{i=-\infty}^{\infty} \lambda(i)=\infty \text {. }
$$

Let $Z$ be the set of all integers and let

$$
X=\prod_{i=-\infty}^{\infty} Z_{i}, \quad Z_{i}=Z, \quad i=0, \pm 1, \cdots
$$

A generic element of $X$ is a point

$$
x=\left\{z_{i}(x)\right\} .
$$

A cylinder of $X$ is a set of the form

$$
C_{m, n}(x)=\left\{y \in X: z_{i}(x)=z_{i}(y), m \leqq i \leqq n\right\} .
$$

Let $B$ be the Borel field generated by the cylinders of $X$ and let $p$ be the $\sigma$-finite measure generated by the cylinder function

$$
p C_{m, n}(x)=\lambda\left(z_{m}(x)\right) \prod_{i=m}^{n-1} p\left(z_{i}(x), z_{i+1}(x)\right) .
$$

It is clear that the measure $p$ is invariant under the shift transformation $T$,

$$
T\left\{z_{i}\right\}=\left\{z_{i}^{\prime}\right\}, \quad z_{i}^{\prime}=z_{i+1},
$$

and $X=\bigcup_{i=-\infty}^{\infty} X_{i}, p\left(X_{i}\right)=\lambda(i), p(X)=\infty$, where $X_{i}=\{x \in X$ : $\left.z_{0}(x)=i\right\}$.

We refer to $(X, \beta, p, T)$ as the $\sigma$-finite stationary Markov chain defined by $P . T$ is the Markov transformation defined by $P$.

We shall be interested in the following conditions on $P$ :

$\mathrm{I}_{k}$. For every $i_{1}, \cdots, i_{k} ; j_{1} \cdots j_{k}$ there exists $n>0$ such that

$$
p^{n}\left(i_{1}, j_{1}\right) \times \cdots \times p^{n}\left(i_{k}, j_{k}\right)>0 .
$$

$\mathrm{II}_{k} . \sum_{n=1}^{\infty}\left[p^{n}(0,0)\right]^{k}=\infty$.

Theorem. $P$ satisfies $\mathrm{I}_{k}$ and $\mathrm{II}_{k}$ if and only if the Markov transformation $T$ defined by $P$ satisfies: $T^{(k)}$ is ergodic with respect to $p^{(k)}$ $=p \times \cdots \times p$ ( $k$ times $)$.

The above theorem can be deduced from a similar theorem in [3]. We indicate below the main points of the proof.

The theorem need only be proved for the case $k=1$. In fact, if

$$
R\left(i_{1}, \cdots, i_{k}\right)=X_{i_{1}} \times \cdots \times X_{i_{k}},
$$

then condition $\mathrm{I}_{k}$ states that 


$$
\lambda^{-1}\left(i_{1}\right) \cdots \lambda^{-1}\left(i_{k}\right) p^{(k)}\left[R\left(i_{1} \cdots i_{k}\right) \cap\left(T^{(k)}\right)^{-n} R\left(j_{1} \cdots j_{k}\right)\right]>0
$$

for some $n>0$. Condition $\mathrm{II}_{k}$ states that

$$
\sum_{n=1}^{\infty}[\lambda(0)]^{-k} p^{(k)}\left[R(0, \cdots, 0) \cap\left(T^{(k)}\right)^{-n} R(0, \cdots, 0)\right]=\infty .
$$

The $k$-dimensional direct product $\left(X^{(k)}, \mathbb{B}^{(k)}, p^{(k)}, T^{(k)}\right)$ of the system $(X, B, p, T)$ can be regarded as 1 -dimensional by relabelling the $k$ vector states $\left(i_{1}, \cdots, i_{k}\right)$ with integers. After relabelling, in view of (2.1) and (2.2) conditions $\mathrm{I}_{k}$ and $\mathrm{II}_{k}$ become $\mathrm{I}_{1}$ and $\mathrm{II}_{1}$.

If $I_{1}$ is not satisfied then for some $(i, j), p^{n}(i, j)=\lambda^{-1}(i) p\left(X_{i} \cap T^{-n} X_{j}\right)$ $=0$ for all $n>0$ and $T$ is not ergodic.

If $\mathrm{II}_{1}$ is not satisfied then

$$
\sum_{n=1}^{\infty} p^{n}(0,0)<\infty,
$$

the state $X_{0}$ is not recurrent [4], and $T$ is not ergodic since a wandering set of positive measure exists [1].

Suppose $I_{1}$ and $I_{1}$ are satisfied, then almost all points of $X_{0}$ return infinitely often to $X_{0}$ under both positive and negative iterations of $T$ and the smallest invariant set containing $X_{0}$ is essentially the whole space $X$ (cf. $[4, \S 4]$ ).

The remainder of the proof can be completed by showing that the transformation induced by $T$ on $X_{0}$ [5], is a Bernoulli transformation. The ergodicity of $T$ then follows from the ergodicity of the induced transformation [5].

3. Examples. Let $-1<\epsilon<1$, and define

$$
Q=\|q(i, j)\|, \quad i=0, \pm 1, \pm 2, \cdots
$$

where $q(i, i+1)=(1-\epsilon / i) / 2, q(i, i-1)=(1+\epsilon / i) / 2, i \neq 0, q(0,1)$ $=q(0,-1)=1 / 2$, and $q(i, j)=0$ if $j \neq i+1$ and $j \neq i-1$.

Let $M=\{m(i)\}, i=0, \pm 1, \cdots$, where

$$
m(0)=1, \quad m(i)=m(-i)=\frac{\Gamma(1+\epsilon) i \Gamma(i-\epsilon)}{\Gamma(1-\epsilon) \Gamma(i+1+\epsilon)}, \quad i>0 .
$$

One can easily verify that

$$
M Q=M .
$$

Let $Q^{2}=\left\|q^{2}(i, j)\right\|$ and put

$$
P=\|p(i, j)\| . \quad i, j=0, \pm 2, \pm 4, \cdots,
$$


where $p(i, j)=q^{2}(i, j)$. Let $\Lambda=\{\lambda(i)\}, i=0, \pm 2, \pm 4$, where $\lambda(i)=m(i)$. Then $\Lambda P=\Lambda$ and $p(i, j)=0$ if and only if $j \neq i-2, j \neq i$ and $j \neq i+2$. $P$ satisfies condition $\mathrm{I}_{k}$ for every $k=1,2, \cdots$. (No difficulties arise from considering matrices $P$ defined over the lattice of pairs of even integers.)

Moreover,

$$
\sum_{i} \lambda(i)=\infty \quad \text { if }-1<\epsilon \leqq \frac{1}{2}
$$

since

$$
\lambda(n) \sim \frac{\Gamma(1+\epsilon)}{\Gamma(1-\epsilon)} n^{-2 \epsilon} .
$$

We shall need the following result of Gillis [2].

Lemma. For any $\theta>0$ there exists $K_{1}=K_{1}(\theta)$ such that for al' $N$,

$$
K_{1}^{-1} N^{\epsilon-1 / 2-\theta}<q^{2 N}(0,0)=p^{N}(0,0)<K_{1} N^{\epsilon-1 / 2+\theta} .
$$

Choose a positive integer $k$ and $\eta>0$ such that

$$
\frac{1}{k}>\frac{1+\eta}{1+k}
$$

Choose $\epsilon$ such that

$$
\frac{1}{2}-\frac{1}{k}<\epsilon<\frac{1}{2}-\frac{1+\eta}{1+k}
$$

and $\theta>0$ such that

$$
\theta<\min \left(\epsilon-\frac{1}{2}+\frac{1}{k}, \frac{1}{2}-\epsilon-\frac{1+\eta}{1+k}\right) ;
$$

then

$$
-\frac{1}{k}<\epsilon-\frac{1}{2}-\theta<\epsilon-\frac{1}{2}+\theta<-\frac{1+\eta}{1+k} .
$$

Consequently, by the lemma, there exists $K_{1}=K_{1}(\theta)$ such that

$$
K_{1} N^{-1 / k}<K_{1} N^{\epsilon-1 / 2-\theta}<p^{N}(0,0)<K_{1} N^{\epsilon-1 / 2+\theta}<K_{1} N^{-(1+\eta) /(1+k)}
$$

i.e.,

$$
\left(p^{N}(0,0)\right)^{k+1}<\left(K_{1}\right)^{k+1} N^{-(1+\eta)}
$$

and 


$$
\left(p^{N}(0,0)\right)^{k}>\left(K_{1}\right)^{k} N^{-1}
$$

Hence, by the theorem, the Markov transformation defined by $P$ has ergodic index $k$.

Finally, if $\epsilon=1 / 2$, then again by the lemma

$$
\sum_{n=1}^{\infty}\left[p^{n}(0,0)\right]^{k}=\infty \quad \text { for } k=1,2, \cdots,
$$

and consequently the Markov transformation defined by $P$ has infinite ergodic index.

\section{REFERENCES}

1. P. R. Halmos, Lectures in ergodic theory, Publications of the Mathematical Society of Japan, no. 3, Mathematical Society of Japan, 1956.

2. J. Gillis, Centrally biased discrete random walk, Quart. J. Math. (2) 7 (1956), 144-152.

3. T. E. Harris and H. Robbins, Ergodic theory of Markov chains admitting an infinite invariant measure, Proc. Nat. Acad. Sci. U.S.A. 39 (1953), 860-864.

4. K. L. Chung, Markov chains with stationary transition probabilities, Springer, Berlin, 1960.

5. S. Kakutani, Induced measure preserving transformations, Proc. Imp. Acad. Tokyo 19 (1943), 635-641.

YALE UNIVERSITY AND

UNIVERSITY OF BIRMINGHAM, ENGLAND 